\title{
Factorization effect and near-threshold boson production at linear colliders
}

\author{
V. Kuksa ${ }^{* a}$, R. Pasechnik, ${ }^{b}$, G. Volchanskiy ${ }^{a}$ \\ ${ }^{a}$ Research Institute of Physics, Southern Federal University, Russia \\ ${ }^{b}$ Department of Physics and Astronomy, Uppsala University, Box 516, SE-751 20 Uppsala, \\ Sweden \\ E-mail: kuksa@list.ru, roman.pasechnik@fysast.uu.se, \\ nikolay.volchanskiy@gmail.com
}

We calculate cross-section of the near-threshold three-boson production at the ILC with an account of their instability and a principal part of NLO corrections. The calculations are fulfilled within the framework of the model of unstable particles with smeared mass. We show that the contribution of finite width (instability) is large in the resonance Higgs-mass range and should be taken into account. Suggested approach significantly simplifies calculations with respect to traditional one.

PACS : $11.30 . \mathrm{Pb}$

The XIXth International Workshop on High Energy Physics and Quantum Field Theory, QFTHEP2010 September 08-15, 2010

Golitsyno, Moscow, Russia

\footnotetext{
* Speaker.
} 


\section{Introduction}

Linear colliders are suitable instrument to study gauge bosons and Higgs physics. The processes of two- and three-boson production give a direct information on trilinear and quartic vector boson coupling [1]. Near-threshold production of the unstable particles (UP's), as a rule, is accompanied by large finite-width effects (FWE). The boson-pair production $e^{+} e^{-} \rightarrow Z Z ; W^{+} W^{-} ; Z H ; Z \gamma$ at threshold energy (LEP II experiments) have been considered analytically with an account of oneloop EW corrections [2] (and references therein). Now, the processes of three-boson production $Z Z Z$ and $Z W^{+} W^{-}$are intensively studied $[3,4]$. These processes are available at Tevatron, $L H C$ and future $I L C$. To describe FWE we have to consider full process $e^{+} e^{-} \rightarrow Z Z Z ; Z W^{+} W^{-} \rightarrow$ $\sum_{f} 6 f$. So, the bosons are considered in the off-shell states and their instability is described by Breit-Wigner propagators. An account of complete NLO corrections to these processes is very complicated problem. Now, the consideration was fulfilled in the stable-particle approximation (SPA), without an account of FWE and for the case of light Higgs $\left(M_{H}=120 \mathrm{GeV}\right.$ and $\left.150 \mathrm{GeV}\right)$.

In this work, we represent the cross-section of the processes $e^{+} e^{-} \rightarrow Z Z Z ; Z W^{+} W^{-}$with an account of FWE and principal part of NLO corrections. We show that FWE is large for the case of $M_{H} \gtrsim 2 M_{W}, 2 M_{Z}$, when the contribution of Higgs diagrams is dominant. So, this effect should be taken into consideration and can be applied as an additional instrument in the search for Higgs boson.

\section{The method of calculation}

The processes $e^{+} e^{-} \rightarrow Z Z Z ; Z W^{+} W^{-}$at the tree level in SPA are described by the set of diagrams, which is represented in Fig.1. Here, $Z Z Z$-production is described by 9 diagrams of the subset $(a),(b)$, and $Z W^{+} W^{-}$-production by 16 diagrams $(a), \ldots,(e)$. The first subset of diagrams (a) has a resonant character, when $M_{H} \gtrsim 2 M_{W}, 2 M_{Z}$, and plays a significant role in the development of Higgs and FWE contributions. Complete NLO corrections are described by additional thousands

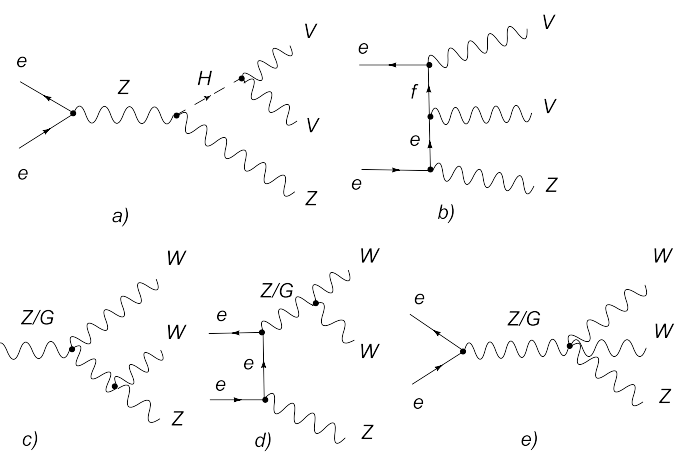

Figure 1: Diagrams for $e e \rightarrow Z Z Z, Z W W$ processes ( $V=Z, W$ and $G$ is photon).

diagrams, which were considered in [3, 4].

To account FWE in a standard way we have to consider full process with 6-particles final states with $Z$ and $W$ in an intermediate state (see Fig.2). However, there is another way of consideration. The model of UP with smeared mass [5] provides the possibility to describe $Z$ and $W$ in the final 
state and simultaneously to account FWE [2, 6]. Such a possibility is closely connected with the effect of factorization which takes place in the framework of the effective theory following from the model. It was shown, that for the processes with UP in time-like intermediate state the amplitude square is exactly factorable:

$$
\begin{aligned}
s=0, \frac{1}{2} & |M|^{2} & =k\left|M_{1}\right|^{2} \cdot\left|M_{2}\right|^{2} ; \\
s=1, \frac{3}{2}, 2, \ldots & J\left(|M|^{2}\right) & =k\left|M_{1}\right|^{2} \cdot J\left(\left|M_{2}\right|^{2}\right) .
\end{aligned}
$$

Here, $s$ is spin of UP, $J(A)$ denotes the integration over the momenta space of the final states. So, we can represent full process of $Z Z Z$ production in a factorized form as it is shown in Fig.2. The

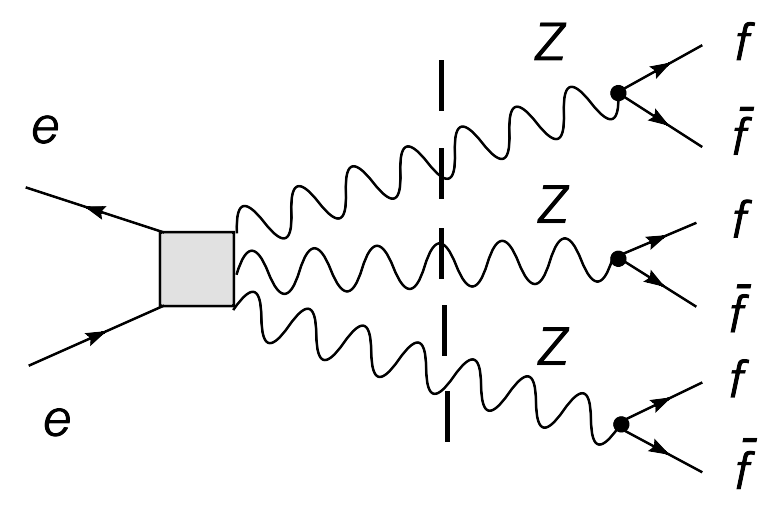

Figure 2: Factorization of $e e \rightarrow Z Z Z$ cross-section.

model cross-section of the inclusive processes $e^{+} e^{-} \rightarrow Z Z Z ; Z W^{+} W^{-} \rightarrow \sum_{f} 6 f$ can be represented in three-convolution form (factorization effect):

$$
\sigma(s)=\iiint \sigma\left(s ; m_{1}^{2}, m_{2}^{2}, m_{3}^{2}\right) \rho\left(m_{1}^{2}\right) \rho\left(m_{2}^{2}\right) \rho\left(m_{3}^{2}\right) d m_{1}^{2} d m_{2}^{2} d m_{3}^{2},
$$

where $\sigma\left(s ; m_{1}^{2}, m_{2}^{2}, m_{3}^{2}\right)$ is Born cross-section for the case of different bosons masses in SPA, $\rho\left(m^{2}\right)$ is probability density of mass square parameter. Born cross-section was calculated with help of the program FeynCalc and integration over variable masses are fulfilled numerically. To escape the singularities, which arise in Higgs-resonance region as a result of integration over phase space of boson pairs, we introduce the $q$-dependent width of Higgs:

$$
\Gamma_{H}(q)=\Gamma_{H}^{s t}(q)+\Gamma_{H}^{W W}(q)+\Gamma_{H}^{Z Z}(q),
$$

where $\Gamma_{H}^{s t}(q)$ is standard width of Higgs at $M_{H}^{2}=q^{2}$ [2] and other two terms account for the bosonpair channels of Higgs decay. It should be noted, that an account of the Higgs width (2.3) is very important because of it forms the results in the resonance region.

The function $\rho\left(\mathrm{m}^{2}\right)$ is given in a traditional Lorentzian form [2, 5]. This approach has a close analogy with the convolution method (CM) [7] (and references therein) and the semi-analytical approximation (SAA) [8], which usually are applied in the frame of standard descriptions. However, the status of these approaches are different (see $[2,5,6])$. In the framework of the model, the expression (2.2) directly follows from the smearing-mass conception for UP and the model function $\rho\left(\mathrm{m}^{2}\right)$ describes the distribution of the mass parameter [5]. Moreover, the model definition of the unstable field function determines a strategy of corrections account $[2,6]$. 


\section{Cross-section of three-boson production}

Firstly, we recalculated the cross-section $\sigma\left(e^{+} e^{-} \rightarrow Z W^{+} W^{-}\right)$at $M_{H}=120 \mathrm{GeV}$ in SPA and with account of FWE (instability of bosons). We have got the results in SPA which coincide with the ones in [3]. The contribution of Higgs diagrams and FWE into full cross-section at $M_{H}=$ $120 \mathrm{GeV}$ occurs small. So, further we have calculated the cross-sections of three-boson production for heavier Higgs.

The cross-section of the process $e^{+} e^{-} \rightarrow Z W^{+} W^{-}$as function of $\sqrt{s}$ at $M_{H}=175 \mathrm{GeV}$ (just above the Tevatron exclusion) is represented in Fig.3. Solid and dashed lines represent Born crosssection in SPA and with account of FWE, dash-point line represents cross-section with ISR corrections which are calculated according to method described in [2]. These corrections are the main part of NLO corrections at the energy range considered. From this picture one can see that the contribution of FWE is large at near-threshold and near-resonant region.

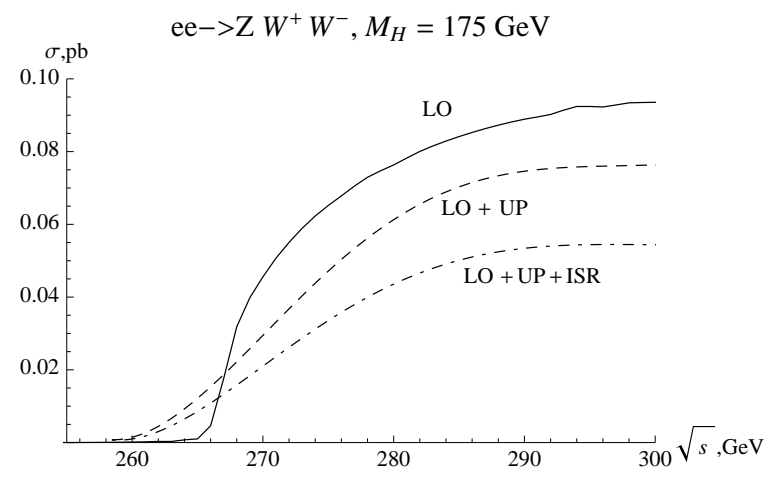

Figure 3: Cross-section of the process $e^{+} e^{-} \rightarrow Z W^{+} W^{-}$.

The cross-section of the process $e^{+} e^{-} \rightarrow Z Z Z$ as function of $\sqrt{s}$ at $M_{H}=195 \mathrm{GeV}$ is represented in Fig.4, where the designations are the same as before. From the picture one can see again that the contribution of FWE is large at near-threshold and near-resonant region.

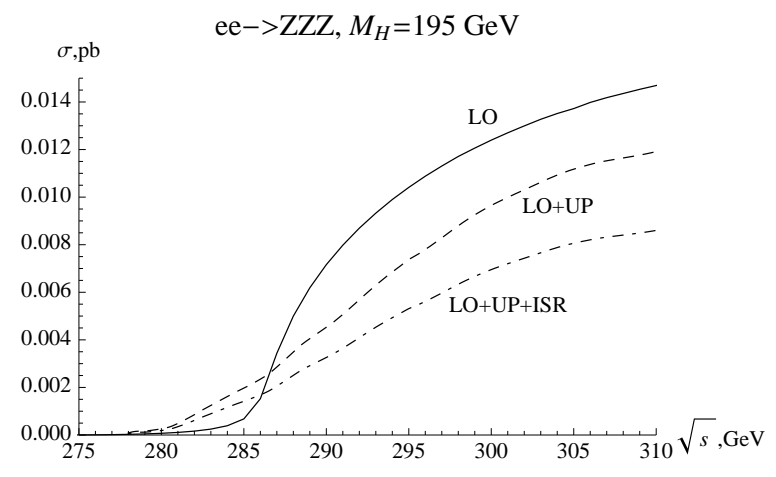

Figure 4: Cross-section of the process $e^{+} e^{-} \rightarrow Z Z Z$.

\section{Conclusions}

From the result of calculations it follows that the value of FWE's strongly depends on Higgs 
diagram contribution. It can be comparable with ISR and one-loop corrections. The contribution of FWE's are large at wide energy range when Higgs-resonant contribution is large and small when Higgs contribution is small. Higgs contribution into cross-section at resonance energy region is strongly dominant. So, Higgs signals in the processes under consideration are large and have well-defined signature. The model approach and factorization effect significantly simplify the calculations in a comparison with the traditional one.

\section{References}

[1] G. Belanger and F. Boudjema, Probing quartic couplings of weak bosons through three vector production at a 500 GeV NLC, Phys. Lett. B 288 (1992) 201.

[2] V.I. Kuksa, R.S. Pasechnik, Near-threshold boson pair production in the model of smeared-mass ustable particles, Phys. Atom. Nucl. 73 (2010) 1622 [hep-ph/ 0910 . 4644 ].

[3] Su Ji-Juan et. al., Complete one-loop electroweak corrections to ZZZ production at the ILC, Phys. Rev. D 78 (2008) 016007 [hep-ph/0807.0669].

[4] F. Boudjema et. al., NLO corrections to $e^{+} e^{-} \rightarrow W^{+} W^{-} Z$ and $e^{+} e^{-} \rightarrow Z Z Z$, Phys. Rev. D 81 (2007) 073007 [hep-ph/0912.4234].

[5] V.I. Kuksa, Finite-Width Effects in the Model of Unstable Particles with a Smeared Mass, Int. J. Mod. Phys. A 24 (2009) 1185 [hep-ph/ 0902 . 2937].

[6] V.I. Kuksa, R.S. Pasechnik, Near-Threshold W-Pair Production in the Model of Ustable Particles with Smeared Mass, Int. J. Mod. Phys. A 24 (2009) 5765 [hep-ph/ 09022857$].$

[7] G. Altarelli, L. Conti, V. Lubicz, The $t \rightarrow W Z$ b decay in the Standard Model:a critical reanalysis, Phys. Lett. B $\mathbf{5 0 2}$ (2001) 125.

[8] D. Bardin et. al., Semi-analytical approach to off-shell W and Z pair production, [hep-ph/9602339].

[9] B. Kilminister, Higgs boson searches at the Tevatron, (to be published in Proc. of ICHEP 2010, July 26, 2010). 